\title{
UNA EXPERIENCIA DE ELABORACIÓN DE UN SIMULADOR CON GEOGEBRA PARA LA ENSEÑANZA DEL MOVIMIENTO PARABÓLICO
}

\author{
Luis Andrés Castillo Brachoo ${ }^{1,4}$ \\ luiscastleb@gmail.com \\ Juan Luis Prieto González ${ }^{2,4}$ \\ juanl.prietog@gmail.com \\ Ivonne C. Sánchez S. 1,4 \\ ivonne.s.1812@gmail.com \\ Rafael Enrique Gutiérrez Araujo ${ }^{3,4}$ \\ rafael.gutierrez0593@gmail.com \\ ${ }^{1}$ Universidade Federal do Pará \\ ${ }^{2}$ Universidad del Zulia \\ ${ }^{3}$ Universidade Federal do $A B C$ \\ ${ }^{4}$ Asociación Civil "Aprender en Red” http://aprenderenred.com.ve/
}

Recibido: 15/09/2019 Aceptado: 24/11/2019

\section{Resumen}

En los últimos años, los profesores han tratado cada vez más de utilizar los recursos didácticos para apoyar sus clases, un hecho que se ha agregado con la llegada de las tecnologías digitales. En este contexto surgió la tendencia de que los profesores se convirtieran en creadores de este tipo de recursos, pero dejando de lado la sistematización y el deber de compartir con sus compañeros la experiencia de desarrollar este tipo de recursos. Por esta razón, el presente trabajo describe una experiencia concreta de elaborar un simulador con el software GeoGebra para estudiar una situación de tiro libre en el fútbol, utilizando nociones de movimiento parabólico. La elaboración del simulador incluye la resolución de siete "tareas de simulación", que destacan los objetos y los procesos matemáticos que justifican las técnicas de construcción utilizadas por los autores. Finalmente, se presentan algunas reflexiones derivadas de la experiencia de desarrollo del simulador, que consideran, por un lado, el conocimiento matemático movilizado en la producción del simulador y, por otro lado, las acciones y capacidades requeridas para tal elaboración.

Palabras clave: Simuladores con softwares dinámicos, tareas de simulación, contenido matemático, movimiento parabólico.

\section{AN EXPERIENCE OF DEVELOPING A SIMULATOR WITH GEOGEBRA FOR THE TEACHING OF THE PARABOLIC MOVEMENT}

\begin{abstract}
In recent years, teachers have increasingly sought the use of didactic resources to support their classes, a fact that has been added with the arrival of digital technologies. In this context, the tendency has emerged that teachers have become creators of this type of resources, but leaving aside the systematization and the duty to share with their peers the experience of developing this type of resources. For this reason, the present work describes a concrete experience of developing a simulator with GeoGebra software for the study of a situation of free kick in football, using notions of parabolic movement. The elaboration of the simulator includes the resolution of seven "simulation tasks", which highlight the objects and mathematical processes
\end{abstract}


that should justify the construction techniques used by the authors. Finally, some reflections derived from the simulator development experience are presented, which consider, on the one hand, the mathematical knowledge mobilized in the production of the simulator and, on the other hand, the actions and capabilities required for such an elaboration.

Keywords: Simulators with dynamic software, simulation tasks, mathematical content, parabolic movement.

\section{UMA EXPERIÊNCIA DE ELABORAÇÃO DE UM SIMULADOR COM GEOGEBRA PARA O ENSINO DO MOVIMENTO PARABÓLICO}

\section{Resumo}

Nos últimos anos, professores têm procurado cada vez mais o uso de recursos didáticos para apoiar suas aulas, fato que foi acrescentado com a chegada das tecnologias digitais. Nesse contexto surgiu a tendência que os professores se tornaram criadores desse tipo de recursos, mas deixando de lado a sistematização e o dever de dividir com seus pares a experiência de desenvolver este tipo de recursos. Por este motivo, o presente trabalho descreve uma experiência concreta de elaboração de um simulador com o software GeoGebra para o estudo de uma situação do tiro livre no futebol, utilizando noções do movimento parabólico. A elaboração do simulador inclui a resolução de sete "tarefas de simulação", que destacam os objetos e processos matemáticos que devem justificar as técnicas de construção utilizadas pelos autores. Finalmente, apresentam-se algumas reflexões derivadas da experiência de desenvolvimento do simulador, que consideram, por um lado, o conhecimento matemático mobilizado na produção do simulador e, por outro lado, as ações e capacidades necessárias para tal elaboração.

Palavras-chave: Simuladores com software dinâmico, tarefas de simulação, conteúdo matemático, movimento parabólico.

\section{Introdução}

Os professores enquanto profissionais da educação procuram, cada vez mais, o uso de recursos e ferramentas para melhorar o processo de ensino e aprendizagem em sala de aula. No geral, esses recursos abrangem os livros didáticos, materiais concretos, entre outros. Entretanto, nos últimos anos as Tecnologias Digitais (TD) tem se tornado em novos recursos para a prática dos professores, os quais têm tido a tendência de construir seus próprios recursos por meio digital (ROJANO, 2014), devido ao fato desses recursos digitais terem um maior impacto nas salas de aula. É por isto, que nas últimas décadas, pesquisadores procuram compreender as vantagens didáticas que essas tecnologias representam para a prática do professor e dos alunos em sala de aula (ORTEGA; MEDELLÍN; MARTÍNEZ, 2010).

Um tipo de recurso elaborado com tecnologias digitais por professores são os simuladores computacionais, na medida em que tais recursos possuem o potencial para produzir aprendizagem sobre situações de fenômenos reais o da vida cotidiana do aluno. Diversas pesquisas afirmam que o uso dos simuladores computacionais por parte dos alunos permite- 
lhes: (i) manipular as variáveis vinculadas ao fenômeno ou situação em estudo, (ii) reconhecer as implicações dessas variações sobre tal fenômeno ou situação, (iii) gerar conjecturas, e (iv) fazer predições sobre o comportamento futuro desse contexto (CATALDI; LAGE; DOMINIGHINI, 2013; HONEY; HILTON, 2011; CONTRERAS; GARCÍA; RAMÍREZ, 2010).

Uma tecnologia digital para a elaboração desses simuladores é o GeoGebra, um software concebido como um aplicativo de matemática dinâmica, de código livre, traduzido em mais de 150 idiomas, e que permite realizar animações e simulações para a mobilização de conteúdos próprios da matemática em sua interface, por meio de suas ferramentas e funcionalidades dinâmicas (BARBOSA, 2013, PRIETO, 2017). Além disso, os professores têm encontrado no GeoGebra também possibilidades para a construção de simuladores computacionais que lhes sirvam de apoio no momento de ensinar ciências naturais, como a física (CERVANTES; RUBIO; PRIETO, 2015; RUBIO; PRIETO; ORTIZ, 2016).

Apesar disso, muitos professores têm seu foco na criação desses recursos e abrem mão da importância da sistematização dessas experiências que podem representar uma fonte significativa para outros professores interessados em preparar seus próprios simuladores com o GeoGebra, ou em realizar alterações nos recursos disponíveis na internet elaborados com esse software. A fim de contribuir com a superação dessa situação, a Associação "Aprender en Red" dedicou-se a sistematizar e socializar as experiências de professores que elaboraram simuladores com o GeoGebra, com o intuito de colocar à disposição da nossa comunidade de professores que ensinam física, uma fonte de informação que possa servir de diretrizes para elaborarem seus próprios simuladores ou alterarem os já existentes.

Nesse sentido, o trabalho descreve uma experiência concreta de elaboração de um simulador com o GeoGebra, com o propósito de mostrar a mobilização do conteúdo de movimento parabólico em um contexto especifico: o tiro livre no futebol. A descrição da experiência enfatiza vários aspectos, nomeadamente: (i) a resolução das chamadas "tarefas de simulação", (ii) a maneira de resolver essas tarefas, e (iii) os argumentos que justificam as ações realizadas durante a resolução das tarefas. Além disso, destacam-se os aspectos teóricos (do conhecimento matemático e/ou físico) e técnicos (relacionados às ferramentas do GeoGebra) das construções. 


\section{Elaboração de Simuladores com GeoGebra}

Básicamente a simulação computacional, também chamada de "simulação digital", refere-se à representação de um fenômeno natural ou artificial que se desenvolve ao longo do tempo, usando tecnologias digitais (BAEK, 2009; KOFMAN, 2000; PUGNALONI, 2008; RODRÍGUEZ; ROGGERO, 2014). Atualmente, os simuladores computacionais são amplamente utilizados em diversos campos científicos e tecnológicos, como em Engenharia, Medicina, Economia, Matemática, Física, entre outros. No campo da Educação, os simuladores computacionais têm sido utilizados como recursos para estudar fenômenos e conteúdos da Matemática e das Ciências Naturais (CLARK; NELSON; SENGUPTA; D’ANGELO, 2009). Ao utilizarem um simulador, os alunos interagem com o modelo computacional do fenômeno associado, de tal forma que conseguem manipular as variáveis que controlam o modelo para analisar, compreender e prever o comportamento real do fenômeno, à medida que o tempo decorre.

Nesse contexto, o simulador elaborado com o GeoGebra é entendido como um modelo computacional que recria uma certa realidade em que um fenômeno físico está subjacente. O simulador pode ser produzido através das ferramentas e funcionalidades dinâmicas do software (CASTILLO; PRIETO, 2018), que permitem reproduzir aspectos do comportamento do fenômeno em condições ideais. Consequentemente, elaborar um simulador com GeoGebra define-se como aquele processo que consiste em resolver um conjunto de tarefas de simulação que estão associadas a "elementos dinâmicos" (vinculados ao modelo do fenômeno ou situação) e "elementos decorativos" que compõem o cenário que serve de contexto para o fenômeno ou situação escolhida (RUBIO, et. al., 2016). Resolver uma tarefa de simulação - correspondente a um determinado elemento dinâmico do contexto escolhido - supõe transitar por um ciclo de modelagem matemática (GUTIÉRREZ; PRIETO; ORTIZ, 2017), o que demanda a construção, no software, de figuras geométricas representativas das formas e movimentos característicos do fenômeno físico que é modelado.

$\mathrm{Na}$ seguinte seção descreve-se a experiência de elaboração de um simulador com GeoGebra para apoiar o ensino do movimento parabólico.

\section{Contexto da experiência}

A experiência da elaboração do simulador, descrita neste trabalho, foi desenvolvida por um professor em formação inicial (de agora em diante, Luiz Aurélio), quem se encontrava 
realizando seu Estágio de Docência III, correspondente ao curso de graduação da Licenciatura em Educação menção Matemática e Física da Universidade do Zulia (Venezuela). Como uma exigência do estágio, o simulador devia ser elaborado de modo que fosse útil para ser usado em uma aula de laboratório de Física com estudantes do quarto ano (16-17 anos) do Ensino Médio. Após a sua preparação, o simulador tinha que ser integrado em um plano de aula para o ensino de algum conteúdo físico.

Dado que não possuam formação alguma no tocante aos aspectos vinculados elaboração desse tipo de simuladores, Luiz Aurélio e seus colegas de estágio participaram em uma oficina sobre elaboração de simuladores com GeoGebra, ministrada pela Associação “Aprender en Red". Tal oficina foi realizada em duas sessões de trabalho (Figura 1).

Figura 1: Oficina de Elaboração de Simuladores com o GeoGebra

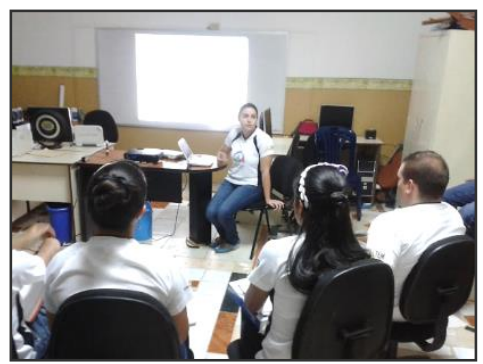

Neste trabalho descrevemos o processo de elaboração do simulador chamado de "Movimento Parabólico no Futebol"1 produzido por Luiz Aurélio após participar da oficina citada. O simulador recria um cenário de práticas de tiro livre de futebol, em que se visualizam tanto a trajetória parabólica de uma bola que é chutada por um jogador, quanto os valores das magnitudes físicas associadas a esse fenômeno. O recurso permite a manipulação de variáveis que definem três magnitudes: as primeiras do fenômeno físico (velocidade e ângulo de tiro), e a terceira pertence à cena que ilustra a distância entre o gol e a bola.

\section{Fenômeno da simulação e considerações}

Conforme mencionado, o fenômeno da simulação que relatamos aqui está relacionado ao movimento parabólico. Tal movimento torna-se evidente quando um projétil é lançado de qualquer superfície, formando um ângulo com a horizontal (BRETT; SUAREZ, 1998). Por projétil entende-se qualquer corpo que se desloque com certa velocidade (SEARS; ZEMANSKY; YOUNG; FREEDMAN, 2004). Normalmente, um projétil com esse movimento

${ }^{1} \mathrm{O}$ simulador pode ser visualizado no seguinte endereço eletrônico: https://ggbm.at/tcbfhz82. 
descreve um percurso curvo que pode ser modelado por uma porção de parábola, desde que o ângulo entre a horizontal e o vetor de velocidade seja agudo (SERWAY; BEICHNER, 2002). O movimento parabólico é considerado como a combinação de um movimento horizontal com velocidade constante (movimento linear uniforme) e uma constante de aceleração vertical (igual à gravidade, aproximadamente $9,8 \mathrm{~m} / \mathrm{s}^{2}$ ). O Quadro 1 mostra as fórmulas matemáticas que descrevem as relações entre as magnitudes presentes neste tipo de movimento.

Quadro 1. Fórmulas associadas ao movimento parabólico

\begin{tabular}{lc}
\hline \multicolumn{1}{c}{ Fórmula } & Rotulagem \\
\hline$V_{o y}=V_{o} \operatorname{sen}(\alpha)$ & Componente da velocidade inicial em $y$ \\
$V_{o x}=V_{o} \cos (\alpha)$ & Componente da velocidade inicial em $x$ \\
$x=V_{o x} t$ & Deslocamento horizontal \\
$y=V_{o y} t-(1 / 2) g t^{2}$ & Deslocamento vertical \\
$t_{\text {max }}=V_{\text {oy }} / g$ & Tempo máximo \\
$t_{\text {voo }}=2\left(t_{\text {max }}\right)$ & Tempo de voo \\
$R=V_{\text {ox }} t_{\text {vuelo }}$ & Alcance máximo \\
\hline
\end{tabular}

Fonte: Adaptado de Bett e Suárez (1998)

Segundo Shirts (1975), a elaboração do modelo correspondente a uma simulação deve basear-se em determinados critérios, regras ou considerações iniciais, que garantam uma representação o mais consistente possível com o comportamento do fenômeno simulado. A partir disso, o desenvolvimento do simulador com o GeoGebra levou em consideração os seguintes aspectos de natureza teórica:

- A trajetória da bola é representada por uma porção de parábola, cuja fórmula é derivada do Quadro 1;

- A aceleração adquirida pela bola em movimento leva o valor da gravidade (g) de $9,8 \mathrm{~m} / \mathrm{s}^{2}$

- Os valores da velocidade e do ângulo de tiro da bola são inseridos por meio do Campo de entrada; 
- A distância entre o gol e a bola é representada por meio de um controle deslizante, que permite que esse valor seja ajustado

\section{Tarefas de simulação e sua resolução}

Para o desenvolvimento do simulador do movimento parabólico, Luiz Aurélio resolveu sete (07) tarefas de simulação, as quais foram abordadas de forma progressiva. Do grupo de tarefas de simulação, quatro delas (tarefas 1,3,4 e 7) tinham vínculo com elementos dinâmicos do simulador, enquanto as tarefas 2, 5 e 6 foram vinculadas com questões próprias da cena. Todas as tarefas foram resolvidas na janela de visualização do GeoGebra 5 clássico, em sua aparência Gráfico, na medida em que as ferramentas dinâmicas oferecidas por essa interface facilitaram a construção e manipulação de modelos matemáticos para a representação do fenômeno. Além disso, Luiz Aurélio optou por utilizar a janela de visualização 2 do GeoGebra como painel de controle, com o intuito de que fosse uma área na interface em que pudessem distribuir-se as variáveis que controlam a trajetória da bola e os gráficos associados. Nas seguintes subseções descreve-se a resolução de cada um dos grupos de tarefas.

\section{Tarefa 1: Representar a trajetória parabólica da bola}

A trajetória da bola foi representada através de uma porção de uma parábola que, para ser definida, foi oportuno elaborar alguns desenhos das possíveis trajetórias pelo movimento da bola. Isto resultou nos três casos seguintes em que a bola: (a) cai antes do gol (Figura 2a), (b) cai dentro do gol (Figura 2b), e (c) passa por cima do gol (Figura 2c).

Figura 2: Desenhos das possíveis trajetórias da bola

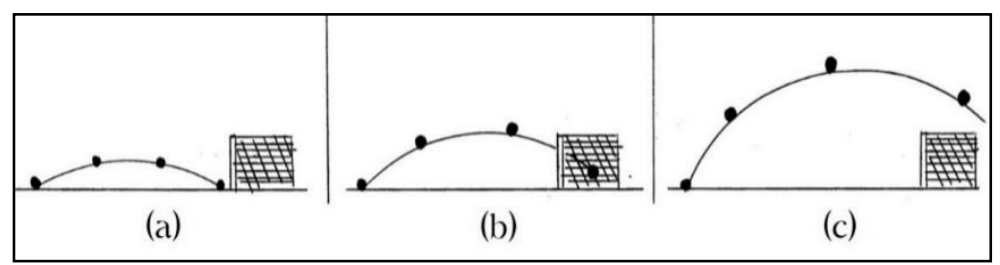

As porções de parábola nos três casos anteriores caracterizam-se por serem côncavas para baixo e conter o vértice. Com base nisso, a tarefa de representar a trajetória da bola consistiu em: (i) estabelecer um modelo matemático (na forma de equação e gráfico) que representasse os três casos de possíveis trajetórias da bola na janela de visualização do GeoGebra, e (ii) 
desenhar a porção de parábola associada ao modelo. A seguir, descreve-se como se atendeu a essas demandas:

\section{Estabelecer o modelo matemático}

Para estabelecer o modelo matemático do fenômeno, foi necessário identificar no Quadro 1 a fórmula que se assemelhava a uma função quadrática, uma vez que o gráfico desse tipo de função é uma parábola. A expressão em questão corresponde à fórmula do deslocamento vertical:

$$
y=V_{o y} t-(1 / 2) g t^{2}
$$

Visto como uma função $y(t)$, a fórmula (1) permite calcular a altura $(y)$ da bola para um determinado tempo $(t)$ do movimento. Inicialmente, não havia informações sobre o tempo da bola em movimento, o que tornava necessário redefinir a fórmula (1), fazendo uma mudança na variável tempo $(t)$ por outras variáveis definidas no início do uso do simulador. A partir da fórmula de deslocamento horizontal, obteve-se que:

$$
t=x / V_{o x}
$$

Substituindo (2) em (1) foi possível obter uma função de altura (y) que não dependia do tempo, mas dependia da distância horizontal $(x)$ percorrida pela bola, conforme mostrado abaixo:

$$
y(x)=V_{o y}\left(\frac{x}{V_{o x}}\right)-\frac{1}{2} g\left(\frac{x}{V_{o x}}\right)^{2}
$$

Percebendo um reorganização dos termos para mostrar a expressão em sua forma padrão, obteve-se o seguinte:

$$
y(x)=-\frac{1}{2}\left(\frac{g}{V_{o x}^{2}}\right) x^{2}+\left(\frac{V_{o y}}{V_{o x}}\right) x
$$

Antes de inserir a fórmula (4) no campo de entrada do GeoGebra, foi necessário criar os valores das variáveis envolvidas nessa expressão, o que levou a:

- Inserir o valor de gravidade $(g)$ no campo de entrada, usando a expressão $g=$ 9.8 .

- Definir a velocidade inicial $\left(V_{o}\right)$ como um controle deslizante de tipo número, chamado $V_{o}$ e com valores mínimo e máximo de 0 e 25 , respectivamente. 
- Criar um controle deslizante de ângulo, chamado $\alpha$, que permitisse definir os componentes da velocidade inicial $\left(V_{o x}\right.$ e $\left.V_{o y}\right)$. Os valores mínimo e máximo desse controle deslizante foram $0^{\circ}$ e $45^{\circ}$.

- Definir as componentes da velocidade inicial $\left(V_{o x}\right.$ e $\left.V_{o y}\right)$ por meio da inserção de $V_{o x}=V o \cdot \cos (\alpha)$ e $V_{o y}=V o \cdot \operatorname{sen}(\alpha)$ no campo de entrada.

Finalmente, a função $f(x)=-\frac{1}{2}\left(\frac{g}{V_{o x}^{2}}\right) x^{2}+\left(\frac{V_{o y}}{V_{o x}}\right) x$ foi inserida no campo de entrada do software para obter então uma parábola côncava para baixo, cujas ramificações se estendem indefinidamente (Figura 3). A decisão de usar a notação $f(x)$ em vez de $y(x)$ responde apenas à necessidade de combinar a expressão com a notação padrão pelo software para as funções de uma variável.

Figura 3: Parábola gerada a partir de $f(x)$

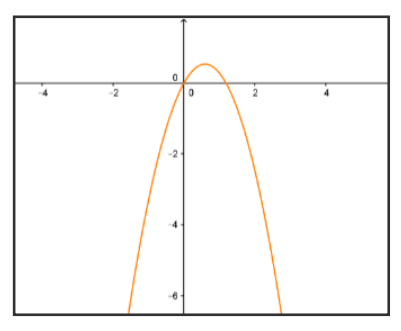

\section{Determinar a porção da parábola}

Para gerar a porção da parábola que representa a trajetória da bola, foi necessário delimitar o domínio da função $f(x)$ originada da fórmula (4). Para tanto, foram consideradas as posições inicial e final da bola no movimento, sendo definidas pelos pontos de coordenadas $(0,0)$ e $(R, 0)$, respectivamente. Na cena, $R$ representa o alcance horizontal máximo da bola, que corresponde ao valor da abscissa do ponto de corte da parábola da Figura 3 com o eixo $x$ à direita da origem do sistema. $R$ foi modelada pela expressão $R=V_{o x} \cdot t_{v o o}$, mostrada no Quadro 1. Antes de gerar o valor de $R$, era necessário definir as variáveis: tempo máximo $\left(t_{\max }\right)$, usando a expressão $t_{\max }=V_{o y} / g$, e tempo de voo $\left(t_{\text {voo }}\right)$, usando a expressão $t_{\text {voo }}=2$. $t_{\max }$. Ambas as expressões foram inseridas no campo de entrada, gerando valores que dependiam das variáveis que os definem. Finalmente, depois de inserir $R$ na barra de entrada, o domínio da função $f(x)$ foi definido da seguinte forma:

$$
\operatorname{Dom} f(x)=\{x \in \mathbb{R} / 0 \leq x \leq R\}
$$


Posteriormente, o gráfico da porção de parábola que modela a trajetória da bola foi obtido a partir da aplicação do comando "Se $(<\operatorname{Condição~}\rangle$, $<$ Então $\rangle)$ " que tem sido útil para a representação com o GeoGebra de funções delimitadas no seu domínio (CONTRERAS, DÍAZ, 2015, RUBIO, PRIETO, ORTIZ, 2016). Para usar esse comando foi necessário definir os parâmetros $<$ Condição $>$ e $<$ Então $>$ para o caso de $f(x)$. Primeiro, o < Condição $>$ refere-se à regra, propriedade ou relacionamento que deve ser atendido para executar um consequente. No caso do simulador, esta condição é dada pelo intervalo $0 \leq x \leq R$, obtido da expressão (5). Segundo, < então > refere-se a toda ação que o software executará se, e somente se, a condição for aplicada. Neste caso, o parâmetro em questão é dado pela expressão que define $f(x)$ (derivado da expressão 4).

A partir desta informação, o comando $\operatorname{Se}\left[0 \leq x \leq R,-\frac{1}{2}\left(\frac{g}{V_{o x}^{2}}\right) x^{2}+\left(\frac{V_{o y}}{V_{o x}}\right) x\right]$ foi inserido no campo de entrada, determinando a porção de parábola que representa os três possíveis casos de trajetória da bola (ver Figura 4).

Figura 4: porção de parábola que modela a trajetória da bola

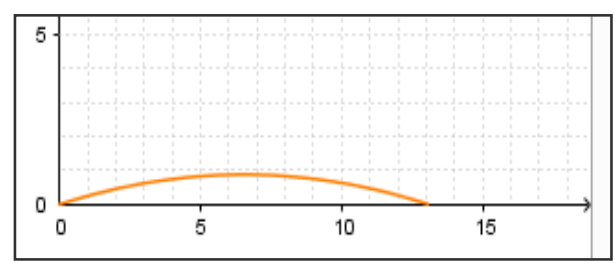

\section{Tarefa 2: Posição do gol na janela de visualização}

O gol foi representado por uma imagem que foi convenientemente inserida na janela de Visualização (ver Figura 5a). Quando uma imagem é inserida no GeoGebra, ela é fixada em dois pontos que são seus cantos inferiores. Conforme permitido pelas restrições impostas a esses pontos, os usuários podem modificar as dimensões da imagem simplesmente arrastando esses elementos pela tela. A fim de evitar deformações subsequentes, a tarefa de posicionar a imagem da meta foi reduzida para determinar três vértices de um retângulo, de modo que os vértices inferiores fossem atribuídos aos cantos correspondentes da imagem e o terceiro vértice servisse para alterar a altura do gol.

O primeiro dos vértices, correspondente ao canto inferior esquerdo da imagem, foi obtido a partir da distância do lance livre mais próximo do gol (11 metros para o pontapé de grande penalidade), tomando cada unidade de comprimento da grade do GeoGebra como um 
medidor de comprimento na realidade. Considerou-se também que esse vértice estaria localizado no eixo $X$, atribuindo o par ordenado $(11,0)$ como suas coordenadas. Esse ponto foi desenhado depois de inserir a expressão $C=(11,0)$ no campo de entrada (ver Figura 5b).

Figura 5: Imagem do gol de futebol e determinação do primeiro vértice

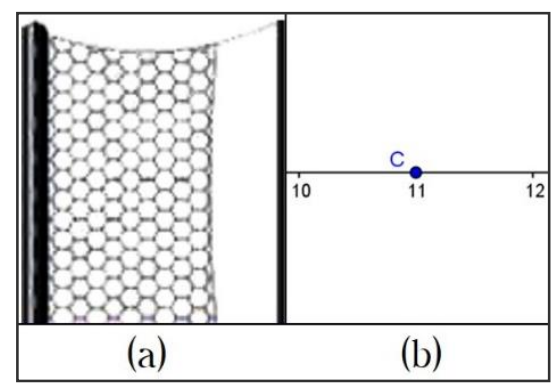

O segundo vértice do retângulo foi mapeado para o canto inferior direito da imagem. Para sua localização, assumiu-se que esse ponto estava no eixo $X$, a alguma distância de $C$. Portanto, a primeira ação realizada foi construir uma circunferência centrada neste ponto de raio 1,5, distancia que corresponde desde um poste da frente ao outro que fica na parte traseira que serve de apoio da rede. Posteriormente, essa curva foi cruzada com o eixo $X$, obtendo o ponto $E$, à direita de $C$ (Figura 6a).

Figura 6: Determinação do segundo e terceiro vértice e inserção da imagem do gol

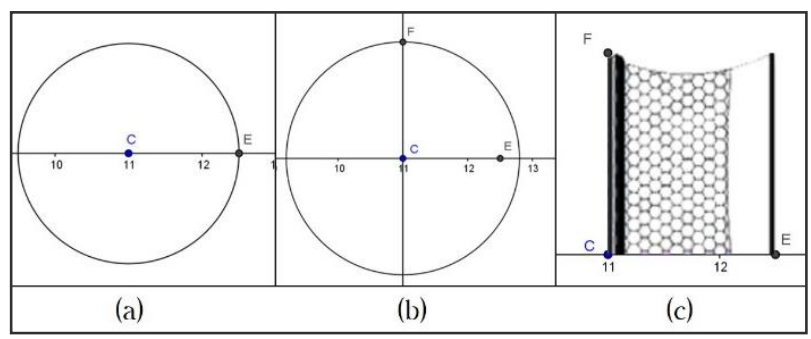

No que diz respeito ao terceiro vértice, foi associado ao canto superior esquerdo da imagem, localizado a certa altura de $C$, em uma linha paralela ao eixo $\mathrm{Y}$ e passando por este ponto. Para localizar esse vértice, foi necessário construir uma circunferência centrada em $C$ de raio 1,8, valor que corresponde à altura por lei do gol, e uma linha paralela ao eixo $Y$ para $C$. A partir da interseção dessas curvas, o ponto $F$ foi obtido (Figura $6 b$ ). Finalmente, a imagem da Figura 5a foi inserida na janela de visualização gráfica, associando seus cantos inferiores com os pontos $C$ e $E$, e o canto superior esquerdo com o ponto $F$ (Figura 6c). 


\section{Tarefa 3: Posicionar a bola na cena}

Conforme foi feito com o gol, a bola foi representada por uma imagem predefinida (Figura 7). No entanto, a diferença dessa tarefa com a anterior reside na necessidade de fazer com que a imagem da bola se deslocara através da janela de Visualização Gráfica de acordo com certas condições. Nesse sentido, a tarefa de posicionar a bola consistiu em: (i) localizar a bola na porção da parábola que descreve a trajetória do seu movimento, (ii) variar a posição da bola em relação ao gol, e (iii) representar o movimento de rotação da bola durante o movimento. A seguir, descreve-se a resolução de cada um desses assuntos:

Figura 7: Imagem da bola de futebol

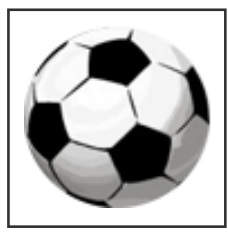

Fonte: https://goo.gl/hqnT2Q

\section{Localização da bola sobre a porção de parábola}

Para localizar a bola na porção de parábola da Figura 3, foi necessário determinar um ponto com a condição de poder deslocar-se ao longo dessa curva com o passar do tempo. Nesse sentido, o primeiro passo foi criar uma variável $R^{\prime}$ que recebe valores entre 0 e $R$. Essa variável foi definida a partir da expressão $R^{\prime}=V_{o x} \cdot t$, em que $t$ foi associado a um controle deslizante de número com um mínimo de 0 e um máximo igual a $t_{v o o}$. Depois disso, a variável $R$ foi criada a partir da inserção de sua expressão no campo de entrada. O segundo passo consistiu em inserir o par ordenado $\left(R^{\prime}, f\left(R^{\prime}\right)\right)$ no campo de entrada, gerando um ponto $F$ na janela de Visualização Gráfica de tal forma que ele se deslocasse através da porção de parábola como $R^{\prime}$ leva valores de 0 a $R$.

Além de localizar $F$ na porção de parábola, foi tomada a decisão de fazer com que esse ponto traçasse a trajetória da bola desde que $R^{\prime}$ tenha valores de 0 a $R$. Para isso, o mesmo comando foi usado conforme na tarefa 1 , substituindo $R$ por $R^{\prime}$, da seguinte maneira: $\operatorname{Se}\left[0 \leq x \leq R^{\prime},-\frac{1}{2}\left(\frac{g}{V_{o x}^{2}}\right) x^{2}+\left(\frac{V_{o y}}{V_{o x}}\right) x\right]$. Dessa forma, uma nova porção da parábola $g(x)$ foi gerada, aparecendo como $F$ deslocado através de $f(x)$ (Figura 8). Para observar esse efeito, foi necessário esconder previamente a porção de parábola da tarefa 1. 
Figura 8: Representando o movimento da bola mediante o ponto $F$

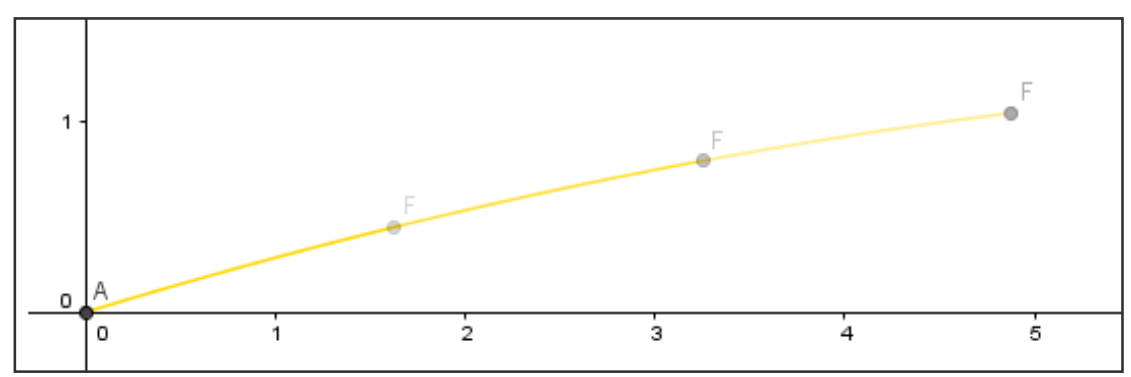

\section{Variar a posição da bola em relação ao gol}

Antes de empreender esta construção, o ponto que indicava a localização da bola era de 11 unidades do gol (distância de tiro de pênalti), conforme ilustra a Figura 9. Entretanto, desejava-se que essa distância aumentasse ou diminuísse de acordo com as necessidades do usuário. Para conseguir isso foi aplicada uma transformação no plano, a translação, tanto para o ponto $F$, quanto para a porção de parábola definida por $g(x)$ (em que $F$ se desloca).

Figura 9: Posição do ponto $\mathrm{F}$ em relação ao gol de futebol

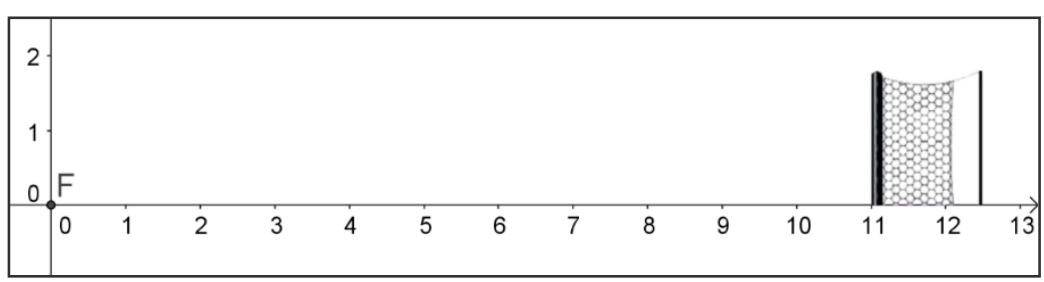

Para aplicar uma translação com GeoGebra é necessário definir o objeto a ser trasladado e um vetor de translação. Desses dois elementos, no momento só se tinham os objetos a serem trasladados, o ponto $F$ e a porção da parábola $g(x)$, portanto, era necessário definir o vetor de translação para cada objeto,. A definição desse vetor significava determinar a direção, módulo e sentido. Como ponto de origem, foi assumido o ponto $A$, localizado na origem do sistema de coordenadas cartesianas. O módulo do vetor foi associado a um controle deslizante de tipo número, chamado $d$, com um valor mínimo de 0,00001 e máximo de 5. Do ponto de vista geométrico, o módulo do vetor foi representado através do diâmetro de uma circunferência centrada em $A$ (Figura 10a) e com raio $d$, mostrando assim o conjunto de pontos do plano que estão a uma distância $d$ da origem do vetor $A$. Alguns desses pontos seriam o extremo do vetor. 
Figura 10: Determinação do vetor $\overrightarrow{A B}$ e translação do ponto $F$ e de $g(x)$

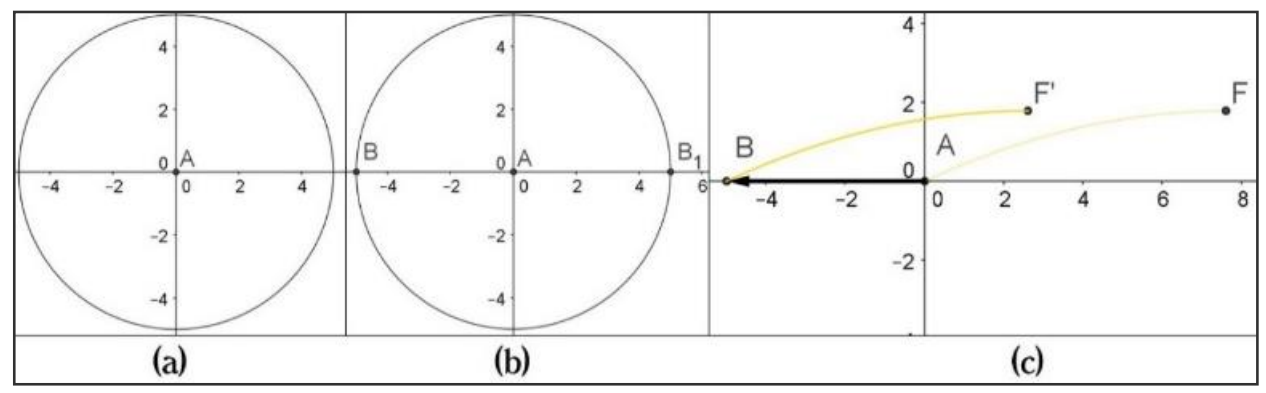

Uma aproximação ao extremo do vetor envolvia levar em conta a direção deste objeto matemático, que era dado pela direção do eixo $X$ (horizontal) de tal forma que, ao intersectar a circunferência e a reta, foram obtidos $B$ e $B_{1}$ (Figura 10b). Como o sentido do vetor era para a esquerda, o vetor de translação foi definido a partir do ponto de corte para a esquerda de A, permitindo a representação do vetor $\overrightarrow{A B}$. Para finalizar, o ponto $F$ e a curva $g(x)$ foram deslocados pelo vetor anterior, obtendo-se os objetos homólogos $F^{\prime}$ e $g(x)^{\prime}$ (Figura 10c).

\section{Representação do movimento de rotação da bola}

Para representar a rotação da bola durante o seu movimento, foi necessário localizar dois pontos de ancoragem da imagem que girava em relação a um ponto localizado no centro da bola. Sabendo que esses pontos poderiam corresponder aos cantos inferiores da imagem, a primeira coisa que foi feita foi construir uma circunferência centrada em $F^{\prime}$ e com raio igual a 0,15 , e uma linha paralela ao eixo $X$ por $F^{\prime}$. Ao cruzar essas curvas, os pontos $G$ e $H$ foram obtidos, os quais, em princípio, foram assumidos como referentes para a localização dos pontos para os cantos inferiores da imagem (Figura 11a). Em outras palavras, $G$ e $H$ indicam a posição dos cantos inferiores da imagem antes de ser girada.

Figura 11: Inserindo a imagem da bola na janela Visualização Gráfica

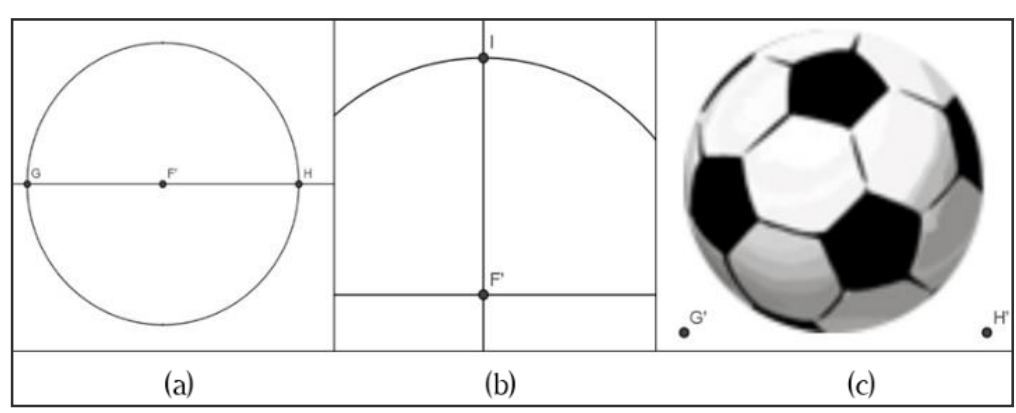

Neste momento, a tarefa de representar a rotação da bola foi reduzida para girar os pontos $G$ e $H$ em relação ao centro da bola, um ponto localizado em uma linha reta perpendicular ao 
eixo $X$ pelo ponto $F^{\prime}$. Era evidente que o centro de rotação poderia ser obtido cruzando a linha e a circunferência previamente construídos, resultando no ponto $I$ (Figura 11b). Para definir o ângulo de rotação, um controle deslizante de ângulo chamado $\beta$ foi criado, com um valor mínimo de $0^{\circ}$ e um valor máximo de $360^{\circ}$. Após a aplicação da rotação nos pontos $G$ e $H$, utilizando o ponto $I$ como centro e o ângulo $\beta$ como ângulo de rotação, foram obtidos os pontos $G^{\prime}$ e $H^{\prime}$, que serviriam para fixar os cantos inferiores da imagem da bola. Finalmente, a imagem da bola foi inserida, ligando os cantos inferiores com esses pontos (Figura 11c).

\section{Tarefa 4: Posicionando o pé do chutador}

Como o gol e a bola, o pé do chutador foi representado por meio de uma imagem previamente selecionada (Figura 12). Para posicionar essa imagem na cena do simulador, foi realizado um procedimento semelhante ao realizado para a tarefa 3.

Figura 12: Imagem do pé do chutador

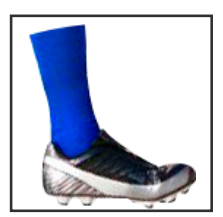

Fonte: https://goo.gl/c172Kc

O procedimento partiu do ponto $B$, o local de onde a bola é colocada em repouso. O primeiro ponto de referência para ancorar a imagem foi obtido cruzando o eixo $X$ com uma circunferência centrada em $B$ e com um raio igual a 0,15 , resultando no ponto $J$. O segundo ponto de referência para a âncora foi obtido ao cruzar o mesmo eixo com uma circunferência com centro em $J$ e raio igual a 0,4 , obtendo o ponto $K$ (Figura 13a).

Figura 13: Inserindo a imagem do pé do chutador na janela de Visualização Gráfica

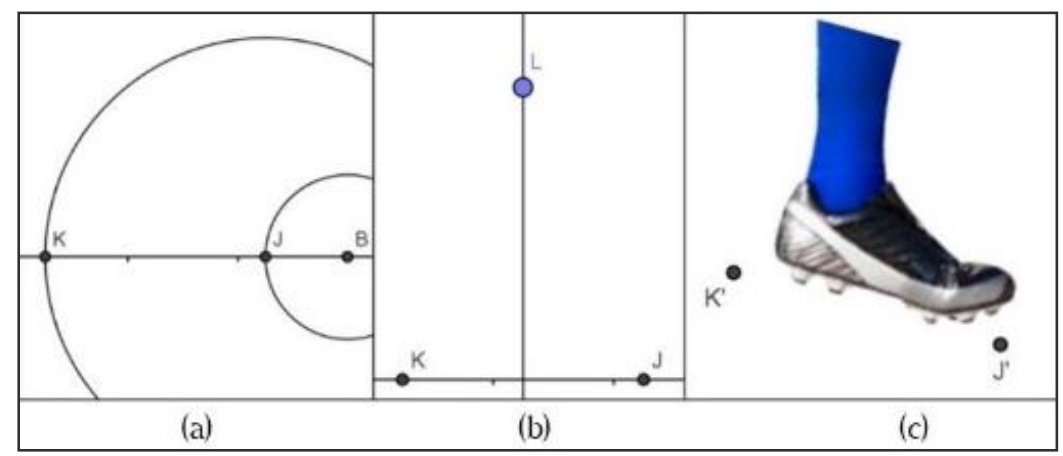


Uma vez que os pontos de referência foram obtidos, eles foram girados de acordo com um centro da rotação e um certo ângulo. O centro de rotação foi localizado a partir da construção de um ponto livre $L$, a mediatriz de $\overline{K J}$. Neste ponto, a propriedade Fixar Objeto foi aplicada para restringir seu movimento (Figura 13b). O ângulo de rotação foi criado por meio de um controle deslizante de ângulo chamado $\gamma$, com um valor mínimo de $0^{\circ}$ e máximo de $45^{\circ}$ (ângulo médio de inclinação do pé do chutador). Finalmente, com esses valores, a rotação foi aplicada aos pontos $K$ e $J$, obtendo os correspondentes homólogos $K^{\prime}$ e $J^{\prime}$, em que os cantos inferiores da imagem do pé do chutador foram fixados (Figura 13c).

\section{Tarefa 5: Representar o céu e o prado da cena}

Para representar o céu e o prado da cena simulada, foi levado em consideração um procedimento de construção com o GeoGebra, usado por Rubio et. al. (2016), para recriar esse tipo de cenários usando como base as inequações. Assim, o céu da cena foi modelado por meio de um semiplano, obtido através da desigualdade $y>0$. Por seu lado, a representação do prado foi conseguida a partir de um semiplano criado com a desigualdade $y<0$. O resultado de ambas as ações é ilustrado na Figura 14.

Figura 14: Construção do céu e prado para a cena simulada

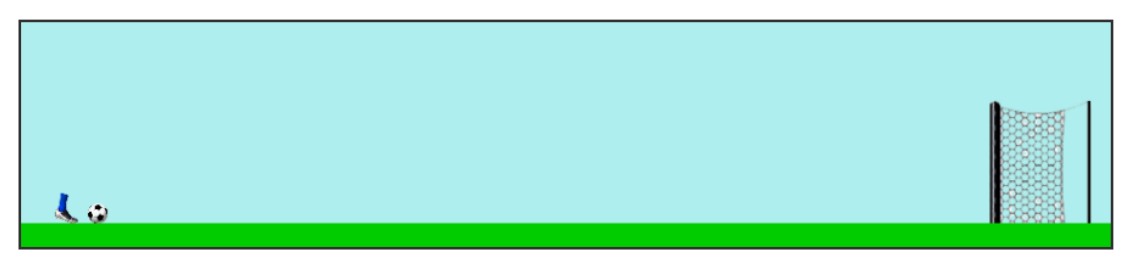

\section{Tarefa 6: Localização do sol, as nuvens e as arquibancadas do estádio de futebol}

$\mathrm{O}$ sol, as nuvens e as arquibancadas do estádio de futebol foram representados por imagens pré-determinadas, que foram inseridas na Vista Gráfica por meio da ferramenta Imagem. É importante ressaltar que, diferentemente do que foi feito nas tarefas anteriores, essas imagens cumpriram um papel meramente decorativo na cena, portanto, o sol, as nuvens e as arquibancadas foram arrastadas pela janela de Visualização Gráfica para que a borda da arquibancada coincidisse com o eixo $X$ e os demais elementos permanecessem acima dos refletores das arquibancadas (Figura 15). Isso permitiu alcançar uma representação de acordo com as características da cena simulada. Finalmente, ao sol, às nuvens e às arquibancadas foi 
aplicada a propriedade Posição Absoluta, a fim de manter sua posição após algum deslocamento ou outra funcionalidade dinâmica.

Figura 15: Posicionamento de aspectos decorativos

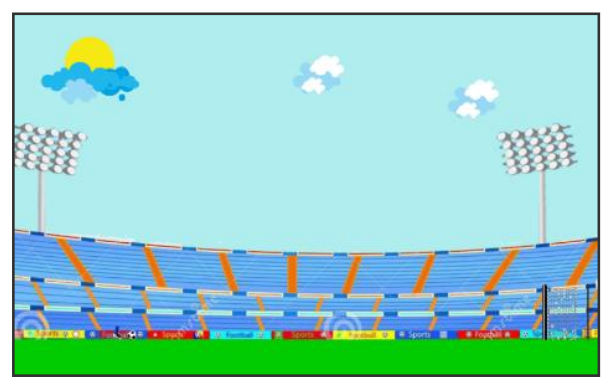

Fonte: https://goo.gl/rR79oM

\section{Tarefa 7: Painel de controle}

Para elaborar o painel de controle, foi necessário dividir a janela de Visualização 2 em duas zonas. Isto foi conseguido inserindo no campo de entrada a expressão $y>6$, cujo semiplano associado foi indicado em amarelo (Figura 16a). Nesta área, duas caixas de entrada e o controle deslizante $d$ foram posicionados, com o objetivo de manipular as variáveis velocidade, ângulo de disparo e distância. Além disso, esses elementos foram acompanhados de textos fixos para identificar os respectivos controles. Na parte inferior da zona, foram posicionados textos dinâmicos que mostram o valor das grandezas físicas ligadas à cena simulada. Finalmente, dois botões foram adicionados, o primeiro chamado "play" que permite iniciar a animação do simulador e o segundo com a função de retroceder as condições na cena (Figura 16b).

Figura 16: Painel de controle do simulador

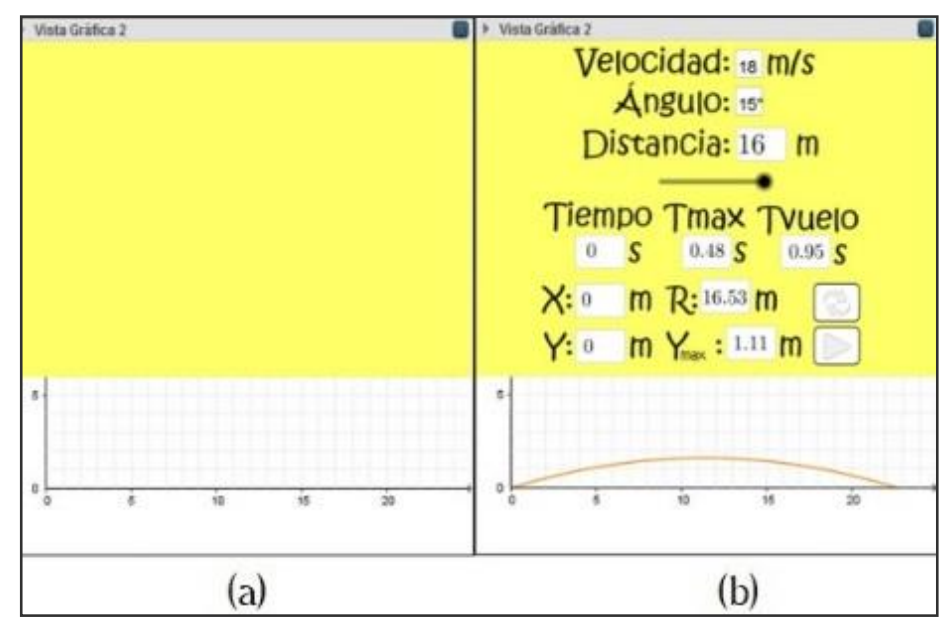




\section{Conclusões}

Neste trabalho foi descrita uma experiência de elaboração de simuladores com GeoGebra, para estudar o movimento de uma bola quando e chutada no futebol desde o tiro livre, em cujo fenômeno encontram-se as ideias do movimento parabólico. Algumas dessas tarefas de simulação foram referidas a elementos dinâmicos, enquanto o restante está associado a elementos decorativos típicos da cena simulada. É importante ressaltar que a resolução de algumas tarefas do primeiro tipo representou um verdadeiro desafio para Luiz Aurélio, na medida em que era a primeira vez que utilizava o software GeoGebra para criar este tipo de recurso digital para o ensino de conteúdos de física.

Para elaborar um simulador que considerasse aspectos tanto do cenário a representar quanto do objeto físico, foi preciso que Luiz Aurélio procurasse: (i) saber mais sobre a cena simulada; (ii) consultar várias fontes de informação sobre a teoria dos projéteis que descrevem os movimentos parabólicos e os modelos matemáticos que sustentam a física para o estudo desse movimento; (iii) refinar os modelos matemáticos gerados; e (iv) usar funcionalidades dinâmicas do GeoGebra, como por exemplos os comandos.

Em primeiro lugar, no tocante à cena simulada, surgiu a necessidade de compreender os elementos constitutivos de uma prática de tiro livre no futebol, entre eles: as dimensões do gol, a distância mínima para que um jogador execute um tiro livre, o intervalo de valores de velocidade e ângulo com o qual uma bola é chutada em condições ideais. Sobre os aspectos físicos dessa situação, vale destacar o entendimento que se deve ter do fenômeno do lançamento do projétil, especialmente o movimento parabólico, suas características básicas e as variáveis de velocidade, ângulo de disparo, deslocamento horizontal ou vertical, tempo, entre outros.

Em segundo lugar, nos modelos matemáticos gerados durante a experiência, é importante destacar a importância da expressão $y(x)=-\frac{1}{2}\left(\frac{g}{V_{o x}^{2}}\right) x^{2}+\left(\frac{V_{o y}}{V_{o x}}\right) x$ para o estabelecimento de um modelo geométrico representativo da trajetória da bola na realidade, o que implicou: (i) um reconhecimento do deslocamento $(y)$ da bola como variável dependente do alcance $(x)$ desta, mas não do tempo $(t)$, como se poderia pensar em uma aula típica de Física; (ii) que a função descrevesse a mudança de posição de um projétil por meio de uma parábola; e (iii) o trabalho algébrico realizado com o domínio da função para determinar o referido traçado da curva. Outros modelos matemáticos, de natureza mais geométrica, surgiram 
precisamente no momento das construções, como foi o caso da translação e da rotação para o momento da tarefa 3.

Finalmente, duas questões relacionadas a determinadas competências-chave para a elaboração de qualquer simulador com o GeoGebra foram reveladas. Por um lado, os diferentes desenhos que foram realizados sobre a cena da simulação mostram a capacidade do sujeito de recriar em sua mente o modelo computacional desejado, com todos os seus elementos constitutivos. Por outro lado, o surgimento de modelos matemáticos utilizados na simulação explica a capacidade de conectar tanto a matemática quanto a física em situações cotidianas e justificar as construções feitas com base nessas conexões. Apesar disso, ainda há muito a ser feito para se obter um melhor entendimento das implicações do desenvolvimento de simulações com o GeoGebra, de forma que este software realmente possa contribuir ao atendimento das necessidades dos professores interessados em elaborar e / ou modificar seus próprios recursos elaborados com tecnologias digitais tais como o GeoGebra.

\section{Referências}

Baek, Youngkyun. Digital Simulation in Teaching and Learning. In: Gibson, D.; Baek Y. (Eds.), Digital Simulations for Improving Education: Learning Through Artificial Teaching Environments, USA: IGI Global, 2009.

Barbosa, S. O software Geogebra e as possibilidades do trabalho com animação. Revista do Instituto GeoGebra Internacional de São Paulo, v.2, n. 1, p.22-32, 2013

Brett, Eli; Suárez, William. Teoría y práctica de Física. Caracas, Venezuela: Corporación Marca, 1998

Cataldi, Zulma; Lage, Fernando.; Dominighini, Claudio. Fundamentos para el uso de simulaciones en la enseñanza. Revista de Informática Educativa y Medios Audiovisuales, v. 10, n.17, p. 8-16, 2013.

Cervantes, Angela; Rubio, Leonela; Prieto, Juan Luis. Una propuesta para el abordaje de la refracción y reflexión total interna utilizando el GeoGebra. Revista do Instituto GeoGebra de São Paulo, v. 4, n.1, p. 18-28, 2015.

Clark, Douglas; Nelson, Brian; Sengupta, Pratim; D’ ANGELO, Cynthia. Rethinking science learning through digital games and simulations: Genres, examples, and evidence. In: The National Research Council Workshop on Gaming and Simulations, Anais..., Washington DC, 2009.

Contreras, Francisco; Díaz, Stepnahie. Elementos de la M16 y la matemática. In: I Encuentro de Clubes GeoGebra del Estado Zulia. Anais..., Maracaibo, Venezuela: A.C. Aprender en Red, 2015.

Contreras, Gloria; García, Rosa; Ramírez, María. Uso de simuladores como recurso digital para la transferencia de conocimiento. Apertura, v. 2, n. 1, 2010. 
Gutiérrez, Rafael; Prieto, Juan Luis; Ortiz, José. Matematización y trabajo matemático en la elaboración de simuladores con GeoGebra. Educación Matematica, v. 29, n. 2, p. 37-68, 2017.

Honey, Margaret; Hilton, Margaret. Learning science through computer games and simulations. National Academies Press, 2011.

Pugnaloni, Luis. Los simuladores. El papel de la simulación en la ciencia. Ciencia Hoy, n. 105, p. 27-34, 2008.

Ortega, G.; Medellín, H.; Martínez, J. Influencia en el aprendizaje de los alumnos usando simuladores de física. Latin American Journal of Physics Education, v. 4, p. 953-956, 2010.

Rodríguez Leonardo; Roggero, Pascal. La modelización y simulación computacional como metodología de investigación social, Polis Revista Latinoamérica, v. 13, n. 39, 2014.

Rojano, Teresa. El futuro de las tecnologías digitales en la educación matemática: prospectiva a 30 años de investigación intensiva en el campo. Educación Matemática, n. 4, p. 11-30, 2014.

Rubio, Leonela; Prieto, Juan Luis; Ortiz, José. La matemática en la simulación con GeoGebra. Una experiencia con el movimiento en caída libre. International Journal of Educational Research and Innovation (IJERI), 2, pp. 90-111, 2016.

Sears, Francis; Zemansky, Mark; Young, Hugo; Freedman, Roger. Física Universitaria Volumen I, $11^{\text {ma }}$ Edición. México: Pearson Educación, 2004

Serway, Raymond; Beichner, Robert. Física para ciencias e ingeniería Tomo I. $5^{\text {ta }}$ Edición, México: McGraw-Hill, 2002.

Shirts, R. Notes on defining simulation. In: Greenblat, C.; Duke, R. (Eds.), Gaming-simulation: Rationale, design and applications. A text with parallel readings for social scientists, educators, and community workers, Toronto: Wiley \& Sons, 1975.

Autores:

\section{Luis Andrés Castillo Bracho}

Es estudiante de la Maestría en Educación en Ciencias y Matemática, adscrita al Programa de Pos-graduación en Educación en Ciencias y Matemática (PPGECM) de la Universidad Federal de Pará (Brasil); es Licenciado en Educación mención Matemática y Física por la la Universidad del Zulia (Venezuela); es Coordinador de Tecnologías Digitales y Soporte de la

Asociación Aprender en Red (http://aprenderenred.com.ve/) y miembro de la Asociación

Venezolana de Educación Matemática (AsoVeMat); ha publicado artículos científicos en revistas arbitradas de Brasil, Colombia y Venezuela; ha participado en eventos del campo de la Educación Matemática realizados en Brasil, Argentina y Venezuela, en calidad de conferencista especial, ponente y tallerista; ha participado como revisor ad-hoc de artículos científicos para revistas de Colombia, Brasil y Venezuela; sus temas de interés actualmente comprenden las construcciones geométricas con software dinámico y usos del GeoGebra en la

enseñanza y aprendizaje de las matemáticas; ha participado en proyectos de investigación tanto en Venezuela, subvencionados por el CONDES-LUZ, como también en proyectos de investigación certificados por el CNPq en Brasil; en 2016 contribuye a que Aprender en Red se adjudique el 2do. lugar del Premio EDUTEC a la Innovación Educativa con TIC 2016 por el Proyecto Club GeoGebra. Email: luiscastleb@gmail.com 
Juan Luis Prieto González

Es estudiante del Doctorado en Educación Matemática de la Universidad de los Lagos (Chile);

Máster en Nuevas Tecnologías Aplicadas a la Educación (Universidad Autónoma de

Barcelona, Universidad de Alicante, Universidad Carlos III de Madrid, España, 2009);

Diplomatura en Estudios Avanzados en la línea de Didáctica de las Matemáticas (Universidad de Alicante, 2008, España); Profesor Agregado de la Universidad del Zulia (Venezuela), encargado de las U. C. Geometría Euclidiana, Geometría Analítica, Practica Profesional I y Practica Profesional III, para la Licenciatura en Educación mención Matemática y Física; es Coordinador General de la Asociación Aprender en Red (http://aprenderenred.com.ve/) y también Secretario de la Asociación Venezolana de Educación Matemática (AsoVeMat); ha publicado libros, capítulos de libro, memorias arbitradas y artículos científicos en revistas arbitradas de España, México, Brasil, Venezuela, Colombia y Chile; sus temas de interés comprenden la formación docente en geometría, aprendizaje del profesor de matemáticas, construcciones geométricas con software dinámico y usos del GeoGebra en la enseñanza y

aprendizaje de las matemáticas; ha participado en eventos del campo de la Educación

Matemática realizados en Venezuela, Colombia, México y Chile, en calidad de conferencista especial, ponente y tallerista; ha dirigido proyectos de investigación subvencionados por el CONDES-LUZ; en 2016 logra que Aprender en Red se adjudique el 2do. lugar del Premio EDUTEC a la Innovación Educativa con TIC 2016 por el Proyecto Club GeoGebra. Email:

juanl.prietog@gmail.com

Ivonne C. Sánchez S.

Es estudiante de la Maestría en Educación en Ciencias y Matemática adscrita al Programa de Pos-graduación en Educación en Ciencias y Matemática (PPGECM) de la Universidad Federal de Pará (Brasil); es Licenciada en Educación mención Matemática y Física por la la

Universidad del Zulia (Venezuela); es la Coordinadora Administrativa de la Asociación Aprender en Red (http://aprenderenred.com.ve/) y miembro de la Asociación Venezolana de Educación Matemática (AsoVeMat); ha publicado artículos científicos en revistas arbitradas de Brasil, España y Venezuela; ha participado en eventos del campo de la Educación Matemática realizados en Brasil, Colombia, Mexico y Venezuela, en calidad de conferencista especial, ponente y tallerista; ha participado como revisora ad-hoc de artículos científicos para revistas de Brasil y Venezuela; sus temas de interés actualmente comprenden las construcciones geométricas con software dinámico y usos del GeoGebra en la enseñanza y aprendizaje de las matemáticas; ha participado en proyectos de investigación tanto en

Venezuela, subvencionados por el CONDES-LUZ, como también en proyectos de investigación certificados por el CNPq en Brasil; en 2016 cotribuye a que Aprender en Red se adjudique el 2do. lugar del Premio EDUTEC a la Innovación Educativa con TIC 2016 por el Proyecto Club GeoGebra. Email: ivonne.s.1812@gmail.com

Rafael Enrique Gutiérrez Araujo

Es estudiante de la Maestría en Enseñanza e Historia de las Ciencias y de la Matemática, adscrita al Programa de Pos-graduación en Enseñanza e Historia de las Ciencias y de la

Matemática (PEHCM) de la Universidad Federal de ABC (Brasil); es Licenciado en Educación mención Matemática y Física por la Universidad del Zulia (Venezuela); es Coordinador de Formación de la Asociación Aprender en Red (http://aprenderenred.com.ve/) y miembro de la Asociación Venezolana de Educación Matemática (AsoVeMat); ha publicado artículos científicos en revistas arbitradas de Brasil, España y México; ha participado en 
eventos del campo de la Educación Matemática realizados en Brasil, Uruguay y Venezuela, en calidad de conferencista especial, ponente y tallerista; ha participado como revisor ad-hoc de artículos científicos para revistas arbitradas de México; sus temas de interés actualmente comprenden la formación de profesores que enseñan matemática y el uso de tecnologías digitales en la enseñanza de la geometría; ha participado en proyectos de investigación tanto en Venezuela (subvencionados por el CONDES-LUZ) como en Brasil (certificados por el CNPq); en 2016 coadyuva a que la Asociación Aprender en Red se adjudique el 2do. lugar del Premio EDUTEC a la Innovación Educativa con TIC 2016 por el Proyecto Club GeoGebra.

Email: rafael.gutierrez0593@gmail.com 